\title{
Special announcement: Guidelines to the Practice of Anesthesia-Revised Edition 2019
}

\author{
Gregory R. Dobson
}

Received: 1 October 2018/Accepted: 1 October 2018/Published online: 11 December 2018

(C) Canadian Anesthesiologists' Society 2018

This issue of the Journal features the 2019 revised edition of the Guidelines to the Practice of Anesthesia ${ }^{1}$ as developed and annually updated by the Canadian Anesthesiologists' Society (CAS) Committee on Standards, and approved by the CAS Board of Directors. The CAS has endorsed Guidelines since their initial development in 1974 (and publication in 1977), and the Journal has published yearly updates since 2009 . They are widely recognized as a valuable clinical resource for anesthesia practitioners both nationally and internationally. Indeed, they are frequently downloaded as free web content and regularly cited in other peer-reviewed articles.

The Committee on Standards welcomes questions, comments, and constructive criticism on this Guidelines document. Any queries and their related responses to them are available on the CAS website. Indeed, this present editorial commentary should be considered an invitation to any anesthesiologist, allied health professional, or healthcare administrator to report their questions or comments to the Committee on Standards (standards@ cas).

In view of the comprehensive and lengthy nature of the Guidelines document, this editorial serves to draw readers' attention to some of the specific topics updated in this new version. It also provides commentary about other issues undergoing further consideration for publication in future versions of the Guidelines.

Appendices to the Guidelines, position papers, and other guideline documents are regularly reviewed by the committee and updated as required. To coincide with the

G. R. Dobson $(\bowtie)$

Committee on Standards, Canadian Anesthesiologists' Society, 1 Eglinton Avenue East, Suite 208, Toronto, ON M4P 3A1,

Canada

e-mail: GDobson@Dal.Ca release of the 2019 revised edition, we are excited to have a comprehensively updated version of Appendix 6, which represents the CAS position paper on procedural sedation. Regular readers of the Journal will have noted that the position paper was published, ${ }^{2}$ along with an accompanying editorial, ${ }^{3}$ in the December 2018 print issue to help achieve a wider circulation and dissemination of the document. For this reason, the CAS felt it deserving of publication on its own, in addition to making it available as electronic supplementary material, which is the usual process for all Guidelines appendices. Readers will notice some important changes in the Guidelines to make them consistent with the new Appendix 6. For example, wherever the term "monitored anesthesia care" appeared in previous editions, it has been replaced by "procedural sedation". In the past, the levels of sedation were described using the modified Ramsey Sedation Scale, from 1 to 6 (where awake, anxious, or restless $=1$ and no response to glabellar tap or loud noise $=6) .{ }^{4}$ It has been replaced by the American Society of Anesthesiologists' Continuum of Depth of Sedation Scale ${ }^{5}$ using the terms minimal, moderate, or deep sedation.

The Standards committee actively considered a variety of topics for revision and implemented a number of changes, which are highlighted in the revised Guidelines. In addition to the material (i.e., content changes) in the Guidelines, the 2019 edition reflects completion of a process initiated with the 2018 edition $^{6}$ to improve some of the language and to achieve more consistency with respect to terminology throughout the document, and thus optimize its readability and clarity. For example, throughout the document, the term "shall" has been replaced by "must". Those types of changes that did not affect meaning are not highlighted in the text. Nevertheless, where a terminology change has significantly changed the meaning or strength 
of the statement, it has been highlighted. An example would be where the term "should" has been changed to "must", making it a much stronger recommendation. The following changes are amongst those implemented and they appear in italics below:

- In section 1.0 (Basic Principles), the importance of continuing medical education and skill maintenance is addressed. The statement has been strengthened and now reads: "All Anesthesiologists must continue their education in the practice of anesthesia, pain management, perioperative care, and resuscitation and participate in a structured maintenance of competence program (e.g., Royal College MOC).”

- The 2018 edition of the Guidelines(6) included new material related to the use of cognitive aids to assist healthcare providers when completing complex decisionmaking and tasks during medical emergencies. It is strongly recommended that healthcare teams participate in simulation or rehearsal of medical emergencies to improve the timeliness and effectiveness of care. As such, in section 3.0 (Anesthetic Equipment and Anesthetizing Location), additional new content reads: "The contents of the (cognitive aid) manual should be regularly reviewed, updated as required, and periodically practiced as a team."

- All Canadians are well aware of the opioid "crisis" in Canada, that it has affected thousands of lives, and that it shows no signs of improving in the short-term. The issues and challenges involved related to street, prescription, and parenteral opioids are complex and diverse. The crisis has focused much needed attention on how we, as physicians and allied health professionals, can show leadership; indeed, many organizations have committed to assisting with finding solutions to some of the problems. ${ }^{7}$ The CAS is committed to looking at ways it can help and strongly encourages a culture of "opioid stewardship" within the specialty. As anesthesiologists, there is much we can do and a good starting point relates to the safe handling, storage, and disposal of controlled medications such as opioids in clinical areas to control access and to minimize the risk of opioid diversion. The Standard's committee has added new content under section 3.1-4 (Responsibilities of the Healthcare Facility) intended to strongly encourage anesthesia departments to become engaged in the issue and to require that they support and implement effective policies including the statements that: "The healthcare facility must ensure that: Through cooperation between the departments of anesthesia and pharmacy there are policies developed and enforced for the safe handling, storage, and disposal of controlled medications (e.g., opioids) in the operating rooms and postanesthesia care unit (PACU) that are consistent with the law, with regulatory authority requirements, and established best practice principles. The goals of such policies should be to limit access of unauthorized personnel to controlled medications, minimize the risk of controlled medication diversion, establish robust documentation procedures that can be audited, while also protecting patient safety by still permitting anesthesia providers immediate access to any medications required for patient care."

- Inhalational anesthesia agents (including nitrous oxide) are greenhouse gases and, as such, have global warming potential. Their impact on the environment will be related to their total annual use and the amount released into the atmosphere from scavenging systems, which will in turn depend on the specific agents that are used and the total fresh gas flow rates utilized while delivering the anesthesia. It is important that anesthesiologists understand the potential environmental impact and do whatever we can to minimize it. The use of lower fresh gas flow rates and preventing the release of agents into the atmosphere from scavenging systems are examples of ways to mitigate the problem. Work is underway to develop equipment and systems to capture and even reuse waste anesthesia gases. Nevertheless, for now, the most effective method we can utilize is to control fresh gas flow rates. Under 3.1-5 (Responsibilities of the Healthcare Facility), it now states: "The healthcare facility must ensure that: Whenever possible, the use of low total gas flow anesthesia is recommended to facilitate the efficient use of inhalational anesthesia agents and to minimize the environmental impact of waste anesthesia agents. Appropriate minimum total gas flows will vary, taking into account the specific inhalational agent and the clinical situation."

- The committee has updated the content specific to the resuscitation of pediatric patients. Section 3.1-7 (Responsibilities of the Healthcare Facility) has been rewritten as follows: "The healthcare facility must ensure that: Equipment appropriate for resuscitating infants and children must be immediately available in any location where sedation or anesthesia involving children is performed. It should also be available in any location receiving pediatric patients who might require resuscitation, such as the emergency department. This equipment must be available to support bag-mask ventilation, endotracheal intubation, fluid administration, temperature monitoring, and advanced cardiac life support. A length-based pediatric emergency tape kit (e.g., Broselow ${ }^{T M}$ ) may facilitate the conduct of resuscitation for providers who infrequently manage pediatric resuscitation."

- Intravenous infusion pump systems have become an integral part of the delivery of anesthesia care. They can be used to deliver a wide variety of medications. To date, the Guidelines have been silent on the importance of the availability of such equipment for the delivery of safe 
anesthesia care. As such, the committee has introduced new content under 3.1-12 (Responsibilities of the Healthcare Facility) as subsection 12 where it states: "The healthcare facility must ensure that: Infusion pump systems with a drug library, appropriate soft and hard limits for each medication, and enabled alarms for the delivery of medications by continuous intravenous infusion are available as required." As stated under subsection 3.114, adequate training on the use of any new anesthesia equipment and documentation that it has been successfully completed is critically important and therefore should be provided for all staff members.

- The 2018 edition of the Guidelines saw the introduction of new recommendations related to minimizing preoperative testing for patients having lowrisk surgery. They also contained new recommendations for preoperative electrocardiograms and chest radiographs for patients having higher risk surgery. The 2019 edition completes the process of revision of the preoperative testing section of the Guidelines by addressing laboratory testing. It also includes a new subsection on the indications for resting echocardiography. It is important for readers to be aware that the laboratory testing recommendations are not for asymptomatic patients having low-risk surgery and that they all start with "may be considered" to emphasize that they do not constitute a definitive list of mandatory test indications; rather, they should help to focus the use of expensive testing only on those patients who will most benefit from them. The changes are too extensive to discuss in detail in this editorial, but they are highlighted in the Guidelines text.

- A minor but important change was made to section $\mathbf{4 . 2}$ (Fasting Guidelines). Enhanced recovery after surgery (ERAS) and other literature has shown that there is an important role for complex carbohydrate beverages in the preoperative phase to help mitigate the deleterious effects of fasting. ${ }^{8}$ They have been added to the list of beverages considered "clear fluids" with the text now reading: "Unless contraindicated, adults and children should be encouraged to drink clear fluids (including water, pulp-free juice, complex carbohydrate beverages, and tea or coffee without milk) up to two hours before elective surgery." Some institutions have been slow to adopt the recommendation of clear fluids up to two hours before anesthesia if not contraindicated. The CAS encourages all institutions to modify their policies to comply with this recommendation. The Standards committee is working on other new changes to fasting guidelines for adults and pediatrics for future Guideline editions.

- In 2016, the International Standards Organization (ISO) ratified a new standard related to connectors used for the delivery of neuraxial medication. Although the new connector intended to replace Luer-type connectors, is not yet in widespread use, nor is it considered mandatory, the committee felt it was important to make readers aware of the ISO standard and to recommend consideration of their use if available. Under section 5.1-6 (Preparation for Anesthesia), it reads: "Before beginning anesthesia, the anesthesiologist must ensure that: If Luer neuraxial connectors are used, both sides of all Luer connectors must be labelled. Consideration should be given to the use of neuraxial connectors complying with ISO 80369-6:2016

- small bore connectors for neuraxial application, (NRFit $\AA$ connectors) subject to availability from equipment suppliers."

- Under section 5.4 (Required Monitoring Equipment), there have been two important changes. Capnography has been required for general anesthesia and deep sedation in previous editions of the Guidelines. This requirement has been extended to moderate sedation in the 2019 edition with the following text: "The following monitoring equipment is required: Capnography for general anesthesia and to assess the adequacy of ventilation for moderate or deep procedural sedation." An entirely new statement appears in the same section as: "The following monitoring equipment must be immediately available: Equipment for invasive hemodynamic monitoring if indicated (e.g, arterial, central venous)."

- Patient monitoring should be considered throughout all the phases of anesthesia care if it is indicated. This recommendation is expressed in a new, more clearly written statement under 6.1 (Recovery Facility). The Standards committee feels strongly that: "Continuous monitoring of patients is recommended during the perioperative period appropriate to the clinical situation. If clinically indicated, supplemental oxygen, portable pulse oximetry, and other appropriate monitoring devices should be applied during transport to the PACU or ICU." Capnography is required in PACU for intubated patients who do not otherwise have disconnect, hypoventilation, or airway obstruction alarm protection such as would be provided with a ventilator. It is also required for patients who are deeply sedated, which is defined as those patients who only respond purposefully following repeated or painful stimulation and therefore ventilation may be inadequate. $^{5}$ The committee has recommended that capnography be considered for unconscious patients with in situ supraglottic devices when removal is not imminent by stating that: "Capnography is required for intubated patients and deeply sedated patients and is recommended for unconscious patients with in situ supraglottic airway devices."

Other topics that the Standards committee are actively discussing for consideration in future editions of the Guidelines include acute pain management and regional anesthesia guidelines, as well as further changes related to 
pediatric patients, fasting guidelines, updating the sections pertaining to obstetrical anesthesia, and enhanced recommendations related to the administration and handling of opioids. We, the CAS Committee on Standards, welcome suggestions for other issues and topics readers feel are appropriately suited to being addressed in our Guidelines.

\section{Communiqué spécial Guide d'exercice de l'anesthésie - Édition révisée 2019}

Ce numéro du Journal présente l'édition 2019 révisée du Guide d'exercice de l'anesthésie ${ }^{1}$ élaborée et mise à jour chaque année par le Comité des normes de pratique de la Société canadienne des anesthésiologistes (SCA) et approuvée par le Conseil d'administration de la SCA. La SCA avalise le Guide depuis sa première rédaction en 1974 (et publication en 1977); le Journal a publié les mises à jour annuelles depuis 2009. Ces lignes directrices sont une ressource clinique importante largement reconnue par les praticiens en anesthésie, autant sur le plan national que sur le plan international. Elles sont fréquemment téléchargées comme contenu Web gratuit et régulièrement citées dans d'autres articles publiés dans des revues à comités de lecture.

Le Comité des normes de pratique accueille avec plaisir les questions, commentaires et critiques constructives concernant ce Guide. Toutes les questions et leurs réponses sont disponibles sur le site Web de la SCA. En fait, cet éditorial-commentaire doit être vu comme une invitation adressée à tous les anesthésiologistes, professionnels de la santé auxiliaires ou administrateurs en soins de santé à faire parvenir leurs questions ou commentaires au Comité des normes de pratique (standards@cas.ca).

Compte tenu du caractère complet et volumineux de ce Guide, cet éditorial a pour but d'attirer l'attention du lecteur sur certains thèmes spécifiques actualisés dans cette nouvelle version. Il propose également un commentaire sur d'autres questions encore débattues qui seront publiées dans les versions ultérieures du Guide.

Les annexes de Guide, les exposés de principe et les autres documents des lignes directrices sont régulièrement révisés par le comité et mis à jour quand cela est nécessaire. Pour coïncider avec la publication de l'édition 2019 révisée, nous sommes heureux d'avoir complètement actualisé la version de l'Annexe 6 qui représente l'exposé de principe de la SCA sur la sédation procédurale. Les lecteurs assidus du Journal auront noté que l'exposé de principe a été publié ${ }^{2}$ avec un éditorial ${ }^{3}$ d'accompagnement dans le numéro imprimé de décembre 2018 pour contribuer à la plus grande diffusion du document. Pour cette raison, la SCA a estimé que cette Annexe méritait une publication à part pour le rendre disponible sous forme de document électronique supplémentaire, ce qui est la procédure habituelle pour toutes les annexes au Guide. Les lecteurs remarqueront aussi certains changements importants dans le Guide pour l'harmoniser avec la nouvelle version de l'Annexe 6. Par exemple, le terme «soins anesthésiques monitorés» présent dans les éditions antérieures a été remplacé par « sédation procédurale » partout où il apparaissait. Par le passé, les niveaux de sédation étaient décrits à l'aide de l'échelle de sédation modifiée de Ramsay, allant de 1 à 6 (où la cote 1 = éveillé, anxieux ou agité et $6=$ une absence de réponse à un stimulus glabellaire (région entre les sourcils) ou à un bruit fort). ${ }^{4}$ Cette échelle a été remplacée par l'échelle de Continuum de profondeur de la sédation ${ }^{5}$ de l'American Society of Anesthesiologists qui utilise les termes de sédation minimale, modérée ou profonde.

Le Comité des normes de pratique s'est activement intéressé à la révision de différents sujets et a implémenté un certain nombre de modifications qui sont soulignées dans la version révisée du Guide. En plus des changements apportés au contenu du Guide, l'édition 2019 témoigne de l'aboutissement d'un processus entamé avec l'édition $2018^{6}$ visant à améliorer la langue utilisée et à parvenir à une plus grande homogénéité pour ce qui concerne la terminologie dans l'ensemble du document, améliorant ainsi sa lisibilité et sa clarté. Ces types de modifications n'ont pas affecté le sens et ne sont pas soulignés dans le texte. Néanmoins, les changements de la terminologie ont été soulignés lorsqu'ils modifiaient significativement le sens ou la force d'un énoncé. Ainsi, l'emploi du conditionnel « devrait » a pu être remplacé par l'indicatif « doit », donnant beaucoup plus de force à cette recommandation. Voici une sélection des changements adoptés qui apparaissent en italiques, ci-dessous :

- Dans la section 1.0 (Principes de base), l'importance de la formation médicale continue et du maintien des compétences est abordée. L'énoncé a été renforcé et voici sa nouvelle version : «Tous les anesthésiologistes doivent continuer leur éducation dans la pratique de l'anesthésie, de la gestion de la douleur, des soins périopératoires et de la ressuscitation en participant à un programme structuré de maintien des compétences (p. ex., MDC du Collège royal). »

- L'édition 2018 du Guide(6) a inclus de nouveaux éléments en rapport avec l'utilisation des aides cognitives pour aider les prestataires de soins de santé lorsqu'ils prennent des décisions et effectuent des tâches complexes au cours d'urgences médicales. Les équipes de soins sont 
fortement invitées à participer à des simulations ou à des répétitions d'urgence médicale pour améliorer l'efficacité des soins et leur rapidité d'exécution. Ainsi, le texte supplémentaire de la section 3.0 (Matériel d'anesthésie et lieux convenant à l'anesthésie), est le suivant: « $L e$ contenu du manuel (aide cognitive) doit être révisé régulièrement, mis à jour selon les besoins et répété de façon périodique en équipe. "

- Tous les Canadiens ont entendu parler de la crise des opioïdes qui a touché des milliers de vies et qui ne montre aucun signe d'amélioration à court terme. Les problèmes et défis impliqués en rapport avec la consommation de rue, sous ordonnance et par voie parentérale des opioïdes sont complexes et variés. La crise a attiré beaucoup d'attention — nécessaire - sur la façon dont nous pouvons faire preuve de leadership en tant que médecins et professionnels de santé associés; de nombreuses organisations se sont engagées à aider à trouver des solutions pour certains de ces problèmes. ${ }^{7}$ La SCA s'est engagée à rechercher les façons d'aider et encourage fortement une culture « de gestion des opioïdes » dans le cadre de la spécialité. En tant qu'anesthésiologistes, nous pouvons faire beaucoup. Un bon point de départ serait la manipulation, le stockage et l'élimination sécuritaires des médicaments contrôlés tels que les opioïdes dans des zones cliniques à accès contrôlé, et de minimiser le risque de détournement des opioïdes. Le Comité des normes de pratique a ajouté un nouveau texte dans une section 3.1-4 (Responsabilités de l'établissement médical) destiné à encourager fortement les services d'anesthésie à s'impliquer dans la problématique et demandé de soutenir et de mettre en œuvre des politiques efficaces incluant des énoncés du type : «L'établissement médical doit s'assurer que : Des règles sont élaborées et appliquées par une collaboration entre les services d'anesthésie et les pharmacies pour la manipulation, le stockage et l'élimination sécuritaires des médicaments contrôlés (p. ex., les opiö̈des) dans les salles d'opération et dans les unités de soins post anesthésie qui soient en conformité avec les lois, les exigences des agences de réglementation et répondent aux principes des meilleures pratiques. Les objectifs de telle politique doivent être de limiter l'accès du personnel non autorisé aux médicaments contrôlés, de limiter le risque de détournement des médicaments contrôlés, de mettre en place des procédures strictes de documentation permettant leur vérification, tout en protégeant également la sécurité des patients en permettant aux prestataires d'anesthésie d'avoir un accès immédiat à tout médicament nécessaire aux soins des patients. »

- Les agents anesthésiques inhalés (dont le protoxyde d'azote) sont des gaz à effets de serre et, en tant que tels, présentent un risque pour le réchauffement planétaire. Leur impact sur l'environnement sera lié à leur utilisation totale au cours de l'année et à la quantité libérée dans l'atmosphère par les systèmes d'évacuation, ce qui dépend évidemment des agents spécifiques utilisés et des débits de gaz frais utilisés pendant l'administration de l'anesthésie. Il est important que les anesthésiologistes comprennent l'impact environnemental potentiel et fassent ce qu'il faut pour le minimiser. L'utilisation de débits des gaz frais plus faibles et la prévention de la libération de ces agents dans l'atmosphère à partir des systèmes d'évacuation sont des exemples de limitation du problème. Des équipements et des systèmes visant à capturer et même réutiliser les gaz anesthésiques rejetés sont actuellement en cours de développement. Cependant, pour le moment, la méthode la plus efficace à laquelle nous pouvons recourir est le contrôle des débits des gaz frais. Il est maintenant écrit dans la section 3.1-5 (Responsabilités de l'établissement médical) : «L'établissement médical doit s'assurer que : autant que possible, l'utilisation de faibles débits de gaz d'anesthésie est recommandée pour faciliter l'utilisation efficace des agents anesthésiques inhalés et pour minimiser l'impact environnemental des agents rejetés à l'extérieur. Le débit global minimum de gaz nécessaire variera en fonction de l'agent inhalé et $d u$ contexte clinique. »

- Le comité a actualisé le contenu spécifique concernant la ressuscitation des patients pédiatriques. La section 3.1-7 (Responsabilités de l'établissement médical) a été réécrite comme suit: "L'établissement médical doit s'assurer que : le matériel nécessaire à la ressuscitation des enfants et des nourrissons doit être immédiatement disponibles en tout lieu où une sédation ou une anesthésie est pratiquée sur des enfants. Il doit être également disponible en tout lieu recevant des patients pédiatriques susceptibles de nécessiter une ressuscitation, tel que le service des urgences. Ce matériel doit être capable de supporter une ventilation au masque, une intubation endotrachéale, l'administration de solutés, le monitorage de la température et la conduite d'une réanimation cardiorespiratoire avancée. Une trousse d'urgence pédiatrique a code couleur basée sur la taille de l'enfant (p. ex., Breslow $\left.{ }^{T M}\right)$ peut faciliter la conduite de la ressuscitation pour les prestataires qui gèrent rarement une ressuscitation pédiatrique. »

- Les systèmes de pompes pour perfusion intraveineuse sont devenus partie intégrante de l'administration des soins anesthésiques. Ils peuvent servir à administrer des médicaments très divers. Jusqu'à maintenant, le Guide est resté muet sur l'importance de la disponibilité d'un tel équipement pour l'administration de soins anesthésiques sécuritaires. Le comité a introduit un nouveau contenu dans la section 3.1-12 (Responsabilités de l'établissement médical), sous-section 12 où il est dit : «L'établissement médical doit s'assurer que: les systèmes de pompes de perfusion avec une bibliothèque de médicaments, les 
limites relatives et absolues pour chaque médicament ainsi que l'activation des alarmes pour l'administration des médicaments en perfusion intraveineuse continue sont disponibles pour répondre aux besoins. " Comme indiqué dans la sous-section 3.1-14, une formation adéquate à l'utilisation de tout nouveau matériel d'anesthésie et à sa documentation doit avoir été complétée avec succès; ceci est d'une importance vitale et doit donc s'appliquer à tous les membres du personnel.

- L'édition 2018 du Guide a vu l'introduction de nouvelles recommandations concernant la limitation des tests préopératoires pour les patients subissant une chirurgie à faible risque. Elle comporte également de nouvelles recommandations pour les électrocardiogrammes et les radiographies du poumon préopératoires chez les patients subissant une chirurgie à risque plus élevé. L'édition 2019 complète le processus de révision de la section sur les tests préopératoires du Guide en abordant la question des examens de laboratoire. Elle inclut aussi une nouvelle sous-section sur les indications de l'échocardiographie au repos. Il est important que les lecteurs comprennent que les recommandations sur les examens de laboratoire ne s'appliquent pas à des patients asymptomatiques subissant une chirurgie à faible risque et qu'elles commencent toutes avec les mots «peut être envisagé » pour insister sur le fait qu'elle ne constitue pas une liste définitive d'indication de tests obligatoires. Il s'agit plutôt d'attirer l'attention sur l'utilisation de tests coûteux à ne pratiquer que chez les patients qui en tireront le plus grand bénéfice personnel. Ces changements sont trop nombreux pour être présentés en détail dans cet éditorial, mais ils sont soulignés dans le texte du Guide.

- Une modification mineure, mais importante, a été apporté à la section 4.2 (Lignes directrices concernant le jeûne). La documentation sur la réhabilitation améliorée après la chirurgie (ERAS) et d'autres articles ont montré que les boissons contenant des hydrates de carbone complexes jouent un rôle important dans la phase préopératoire pour contribuer à limiter les effets délétères du jeûne. ${ }^{8}$ Ils ont été ajoutés à la liste des boissons assimilées aux «liquides clairs », avec le texte suivant: «Sauf contre-indications, les adultes et enfants doivent être encouragés à boire des liquides clairs (incluant l'eau, les jus de fruits sans pulpe, les breuvages contenant des hydrates de carbone complexes, le thé ou le café sans lait) jusqu'à deux heures avant une chirurgie programmée. » Quelques établissements ont été lents à adopter la recommandation sur la prise des liquides clairs jusqu'à deux heures avant une anesthésie en l'absence de contre-indications. La SCA encourage tous les établissements à modifier leurs règles pour se conformer à cette recommandation. Le Comité des normes de pratique travaille sur d'autres changements concernant les lignes directrices sur le jeûne des patients adultes et pédiatriques dans les éditions ultérieures du Guide.

- En 2016, l'Organisation internationale de normalisation (ISO) a ratifié une nouvelle norme concernant les connecteurs utilisés pour l'administration de médicaments par voie neuraxiale. Même si le nouveau connecteur destiné à remplacer le raccord de type Luer n'est pas encore très répandu ni jugé obligatoire, le comité a estimé qu'il était important de communiquer la norme ISO aux lecteurs du Journal et de recommander d'envisager son utilisation, s'il est disponible. Dans la section 5.1-6 (Préparation à l'anesthésie), on peut lire : «avant de commencer l'anesthésie, l'anesthésiologiste doit s'assurer que : si des connecteurs Luer neuraxiaux sont utilisés, les deux côtés des raccords Luer doivent être étiquetés. Il faut envisager l'utilisation de connecteurs neuraxiaux conformes à la norme ISO 80369-6:2016 connecteurs de petit diamètre interne pour application neuraxiale (raccords NRFit $\left.{ }^{\circledR}\right)$ en fonction des disponibilités des fournisseurs de matériel. »

- Il y a eu deux changements importants dans la section 5.4 (Matériel de monitorage requis). $\mathrm{La}$ capnographie a été requise pour une anesthésie générale et la sédation profonde dans les versions précédentes du Guide. Cette exigence a été étendue à la sédation modérée dans l'édition 2019 édition avec le texte suivant : «Le matériel de monitorage suivant est requis : capnographie pour anesthésie générale et pour évaluer le caractère adéquat de la ventilation au cours d'une sédation procédurale modérée ou profonde.» Un énoncé totalement nouveau apparaît dans la même section : «Le matériel suivant doit être immédiatement disponible: Matériel pour monitorage hémodynamique invasif si indiqué (p. ex., ligne artérielle, cathéter veineux central). »

- Le monitorage des patients doit être envisagé pendant toutes les phases des soins anesthésiques en cas d'indication. Cette recommandation est exprimée dans un nouvel énoncé rédigé plus clairement dans la section 6.1 (La salle de réveil). Le Comité des normes de pratique a émis la forte opinion que : «Le monitorage continu des patients est recommandé pendant la phase périopératoire en fonction de la situation clinique. Si cliniquement indiqué, de l'oxygène supplémentaire, une oxymétrie de pouls portable et d'autres dispositifs de monitorage adaptés doivent être utilisés pendant le transfert vers la salle de réveil ou l'USC. » $\mathrm{La}$ capnographie est requise en salle de réveil pour les patients intubés qui ne sont pas autrement reliés à une alarme protectrice de déconnexion, d'hypoventilation ou d'obstruction des voies respiratoires comme en fournirait un ventilateur. Cela est également requis pour les patients sous sédation profonde, c'est-à-dire les patients qui ne répondent de manière adaptée qu'après une stimulation répétée ou douloureuse et chez qui la ventilation pourrait être 
inadéquate. ${ }^{5}$ Le Comité a recommandé que la capnographie soit envisagée pour les patients inconscients avec dispositifs supraglottiques in situ dont le retrait n'est pas imminent, en déclarant : «La capnographie est requise pour les patients intubés sous sédation profonde et est recommandée pour les patients inconscients ayant des dispositifs supraglottiques in situ des voies aériennes. »

Les autres sujets que le Comité des normes de pratique discute activement en envisageant de les inclure dans les éditions ultérieures du Guide incluent la gestion de la douleur aiguë et les lignes directrices d'anesthésie régionale, ainsi que des changements supplémentaires concernant les patients, pédiatriques, les lignes directrices sur le jeûne, la mise à jour de sections portant sur l'anesthésie obstétricale et l'amélioration des recommandations liées à l'administration et à la manipulation des opioïdes. En tant que Comité des normes de pratique, nous serons heureux de recevoir les suggestions des lecteurs sur d'autres problèmes qu'il conviendrait d'aborder dans notre Guide.

Conflicts of interest None declared.

Editorial responsibility This submission was handled by Dr. Hilary P. Grocott, Editor-in-Chief, Canadian Journal of Anesthesia.

Conflits d'intérêts Aucun déclaré.

Responsabilité éditoriale Cet article a été traité par le $\mathrm{D}^{\mathrm{r}}$ Hilary $\mathrm{P}$. Grocott, Rédacteur en chef, Journal canadien d'anesthésie.

\section{References}

1. Dobson G, Chow L, Flexman A, et.al. Guidelines to the Practice of Anesthesia-Revised Edition 2019. Can J Anesth 2019; 66: DOI: https://doi.org/10.1007/s12630-018-1248-2.

2. Dobson G, Chong MA, Chow L, et.al. Procedural sedation: a position paper of the Canadian Anesthesiologists'Society. Can J Anesth 2018; DOI: https://doi.org/10.1007/s12630-018-1230-z.

3. Dobson GR. Special announcement-procedural sedation: a position paper of the Canadian Anesthesiologists' Society. Can J Anesth 2018; DOI: https://doi.org/10.1007/s12630-018-1231-y.

4. Ramsey MA, Savege TM, Simpson BR, Goodwin R. Controlled sedation with alphaxalopne-alphadolone. Br Med J 1974; 2: 656-9.

5. American Society of Anesthesiologists. Continuum of Depth of Sedation: Definition of General Anesthesia and Levels of Sedation/Analgesia. Committee of Origin: Quality Management and Departmental Administration. Available from URL: http://www.asahq.org/ /media/ sites/asahq/files/public/resources/standards-guidelines/continuum-ofdepth-of-sedation-definition-of-general-anesthesia-and-levels-ofsedation-analgesia.pdf (accessed October 2018).

6. Dobson G, Chong M, Chow L, et al. Guidelines to the Practice of Anesthesia-Revised Edition 2018. Can J Anesth 2018; 65: 76104.

7. Joint Statement of Action to Address the Opioid Crisis. Health Canada, 2016. Accessed: July 5, 2018. Available from URL: http:// www.ccsa.ca/Resource\%20Library/CCSA-Joint-Statement-of-ActionOpioid-Crisis-Annual-Report-2017-en.pdf (accessed October 2018).

8. Miller TE, Roch AM, Mythen M. Fluid management and goaldirected therapy as an adjunct to Enhanced Recovery After Surgery (ERAS). Can. J Anesth 2015; 62: 158-68. 